\title{
Editorial
}

Nephrology

\section{Albuminuria: A Novel Marker for Functional Impairment in Older Adults?}

\author{
Michael Heung \\ Department of Medicine, Division of Nephrology, University of Michigan, Ann Arbor, MI, USA
}

Over the past 2 decades, the clinical significance of albuminuria has been increasingly recognized, most notably as an independent predictor of adverse cardiovascular and renal outcomes $[1,2]$. The kidneys can thus be regarded as providing a window on systemic endothelial function, with urinary albumin being a sensitive, inexpensive, and widely available lens through which vascular health can be gauged.

Now, there may be even more reason to pay close attention to urinary albumin excretion. In this issue of the American Journal of Nephrology, Wolfgram et al. [3] present a secondary analysis from the Systolic Blood Pressure Intervention Trial (SPRINT). Using a subgroup of adults aged 75 years or older, they examined data from the baseline study assessment period. In addition to renal indices (estimated glomerular filtration rate [eGFR] and albuminuria), participants in this subgroup underwent functional performance evaluation using 3 different validated tools: (1) gait speed assessment, (2) a self-administered health-related quality of life survey focusing on functional status (EuroQol 5 dimensions questionnaire [EQ-5D]), and (3) a self-administered fall risk assessment tool (Falls Efficacy Scale [FES]). In unadjusted modeling, both decreasing eGFR and increasing albuminuria categories were associated with worse scores on the 3 performance outcome measures. In adjusted models (including adjustment for albuminuria), eGFR was no longer a significant predictor of performance scoring. However, albuminuria remained a significant predictor for both gait speed and EQ-5D, although not for FES scoring.

\section{KARGER}

(๑) 2017 S. Karger AG, Basel

E-Mail karger@karger.com

www.karger.com/ajn
Previous studies have well-characterized the association between advanced renal impairment and decline in functional status, particularly in the end-stage renal disease population [4]. However, few studies have examined this relationship at earlier stages of chronic kidney disease (CKD) or attempted to incorporate albuminuria into the assessment. Recently, Bowling et al. [5] examined fall risk in a cohort of 8,744 adults (including 1,604 adults with CKD) from the Reasons for Geographic and Racial Differences in Stroke study. Similar to the Wolfgram study, decline in eGFR was not associated with increased risk for serious fall injury, whereas fall risk increased with progressively higher category of albuminuria. Together, the findings from the Bowling and Wolfgram studies suggest that albuminuria may be more sensitive than eGFR in identifying patients at risk for functional impairment, at least in patients with earlier stages of CKD. The findings from these studies are both congruent and complementary. While the Bowling study focused on the hard outcome of serious fall-related injury, the Wolfgram study examined surrogates for functional impairment. The latter finding is particularly important because it lends support to the ability to identify a signal for fall risk before an actual event. Falls and fallrelated injuries are highly prevalent and potentially devastating complications in older adults [6], and patients with CKD are at particularly high risk [5]. The ability to identify patients at risk at an early stage - when preventative interventions are most likely to be effective - is therefore an important public health goal.

Michael Heung, MD, MS

Department of Medicine, Division of Nephrology, University of Michigan 1500 E. Medical Center Drive, SPC 5364

Ann Arbor, MI 48109-5364 (USA)

E-Mail mheung@med.umich.edu 
Because of the cross-sectional design of the Wolfgram study, a causative relationship between albuminuria and functional status impairment cannot be inferred. However, as discussed by the authors, there is at least biologic plausibility to link the finding of albuminuria with development of factors that could contribute to impaired functional status. These factors include cardiovascular disease, cerebrovascular disease, and sarcopenia. As the final data from SPRINT become available, future analyses will be able to assess the longitudinal relationship between renal function, albuminuria, and changes in functional status.

In the meantime, from a clinical perspective, one can argue that understanding the exact cause-effect relationship between albuminuria and functional status is less important than recognizing the association itself. At a minimum, Wolfgram et al. [3] have demonstrated that increasing albuminuria is a marker for worse performance on validated measures of functional status in older adults. Furthermore, patients with albuminuria did not appear to rate themselves as being at statistically higher risk for falls (based on the FES). As such, albuminuria appears to be even more sensitive than self-reporting for early changes in functional performance. It would be a reach to use the results of the current study to advocate for albuminuria testing as a screening tool for functional impairment. However, when albuminuria is identified, clinicians should be mindful of the association with functional status and consider the need for further evaluation for subclinical functional impairment.

An important caveat to interpreting the results of this study is to be aware of the relatively strict study inclusion criteria. Patients with diabetes and cerebrovascular dis- ease, who are already at higher risk for functional impairment, were excluded from SPRINT. Similarly, there was incomplete data regarding potential cognitive impairment in the study population (e.g., dementia, Parkinson's disease). Absence of albuminuria in these populations should not be interpreted as a protective factor against functional impairment, and patients should continue to be screened if other clinical risk factors are present. Rather, albuminuria may be most helpful in lower risk populations where the risk is not as readily apparent. In these situations, the finding of albuminuria is not confirmatory in of itself, but should rather be used to risk stratify patients for more formal functional status evaluation. Indeed, considering the relatively modest differences in assessment scores (EQ-5D and gait speed) between albuminuria groups, albuminuria is likely to be a fairly non-specific marker. Patient performance on validated functional screening tests can then be used to determine the need for clinical interventions such as physical therapy vs. follow-up monitoring.

The findings from this study require further validation, particularly in broader patient populations. Nevertheless, Wolfgram et al. [3] have identified albuminuria as a novel independent risk factor for functional impairment in older adults. Given the major morbidity and mortality attributed to fall injuries in this population, any additional information to help risk stratification should be regarded as a welcome sign.

\section{Disclosure Statement}

I have no relevant conflicts of interest or financial disclosures.

\section{References}

1 Pontremoli R, Leoncini G, Ravera M, Viazzi F, Vettoretti S, Ratto E, et al: Microalbuminuria, cardiovascular, and renal risk in primary hypertension. J Am Soc Nephrol 2002; 13(suppl 3):S169-S172.

2 Kidney Disease: Improving Global Outcomes (KDIGO) CKD Work Group: KDIGO 2012 clinical practice guideline for the evaluation and management of chronic kidney disease. Kidney Int Suppl 2013;3:1-150.
3 Wolfgram DF, Garcia K, Evans G, Zamanian S, Tang R, Wiegmann T, Sharma R, Campbell R, Whittle J; the SPRINT Study Research Group: Association of albuminuria and estimated glomerular filtration rate with functional performance measures in older adults with chronic kidney disease. Am J Nephrol 2017;45:172-179.

4 Kurella Tamura M, Covinsky KE, Chertow GM, Yaffe K, Landefeld CS, McCulloch CE: Functional status of elderly adults before and after initiation of dialysis. N Engl J Med 2009; 361:1539-1547.
5 Bowling CB, Bromfield SG, Colantonio LD, Gutierrez OM, Shimbo D, Reynolds K, et al: Association of reduced eGFR and albuminuria with serious fall injuries among older adults. Clin J Am Soc Nephrol 2016;11:1236-1243.

6 Verma SK, Willetts JL, Corns HL, MarucciWellman HR, Lombardi DA, Courtney TK: Falls and fall-related injuries among community-dwelling adults in the United States. PLoS One 2016;11:e0150939. 